\title{
Why We Should Consider Evidence-Based Treatment Options for Truncal Acne
}

Linda Stein Gold · Thomas Dirschka

Received: March 8, 2021 / Published online: April 19, 2021

(C) The Author(s) 2021

Keywords: Truncal acne; Acne; Acne vulgaris; Trifarotene; Sarecycline; Topical treatment; Topical retinoid

\section{Stein Gold}

Clinical Research Division, Head of Dermatology,

Henry Ford Hospital, Detroit, MI, USA

T. Dirschka $(\bowtie)$

CentroDerm Clinic, Heinz-Fangman-Straße 57, 42287 Wuppertal, Germany

e-mail: t.dirschka@centroderm.de

T. Dirschka

Faculty of Health, University of Witten-Herdecke, Witten, Germany

\section{Key Summary Points}

Truncal acne has historically been overlooked in clinical evaluations and trials, resulting in a lack of evidence-based treatment options

Despite the lack of randomized clinical trials and treatment guidelines involving the trunk, we have typically relied on benzoyl peroxide washes, oral antibiotics, and isotretinoin for truncal acne treatment

Some progress has been made in recent years with certain trials including truncal acne evaluation, for example lidoseisotretinoin, sarecycline, dapsone, and trifarotene

Trifarotene is the first topical retinoid with robust evidence from large-scale, randomized, vehicle-controlled phase III studies, supporting its use as a first-line acne treatment for both the face and trunk

It is time we consider evidence-based treatments for our patients with combined facial and truncal acne, to help improve outcomes and relieve the burden on their lives 


\section{DIGITAL FEATURES}

This article is published with digital features, including a summary slide, to facilitate understanding of the article. To view digital features for this article go to https://doi.org/10.6084/ m9.figshare.14356220.

\section{COMMENTARY}

Truncal acne (back and/or chest) is a common presentation of acne vulgaris, affecting approximately $50 \%$ of people with facial acne [1]. However, truncal acne has historically been overlooked in both clinical evaluations during consultations and clinical trials, resulting in a lack of evidence-based treatment options. Indeed, the treatment landscape has remained largely the same for many decades. We have typically relied on benzoyl peroxide (BPO) washes, oral antibiotics, and isotretinoin for truncal acne treatment, despite the lack of randomized clinical trials and treatment guidelines involving the trunk [1]. In the hope of providing better outcomes for our patients, we must avoid inferring efficacy and tolerability on the trunk from facial acne data and look towards gaining robust evidence. Here, we will explore different treatments available for the trunk, including sarecycline and trifarotene, and discuss important considerations for the treatment of truncal acne.

Current treatment guidelines for acne management have limited guidance for the trunk, and thus we continue to manage acne on the back, shoulders, and chest, on the basis of guidelines specifically for facial acne [1]. In our experience, many dermatologists consider systemic therapy a necessary part of the treatment regimen owing to the involvement of large body surface areas in truncal acne. Moreover, there can be some reluctance to use topical agents as a first-line treatment for truncal acne due to the perception of limited efficacy and potential for irritation with retinoids on the trunk, and bleaching effects of BPO on clothing and bed linen.
Systemic therapy like oral isotretinoin, particularly the lidose formulation with some evidence on the trunk, is a valid option for severe inflammatory and nodular acne for those willing to use it [2]. However, a combination regimen involving fixed combinations of topical agents (e.g., retinoid/BPO or clindamycin/BPO) or topical therapies plus systemic antibiotics is generally recommended for other cases [1]. In fact, for patients who present with mild or moderate truncal acne, topical monotherapy may be adequate as both initial and maintenance treatment [1]. Topical treatment on extensive and hard-to-reach areas such as the back and shoulders can be challenging for patients; thus, wash-off formulations of BPO are often recommended for convenient application in the shower and to minimize bleaching/discoloration of clothing and bedsheets [3]. However, the limited clinical studies available demonstrate that the very brief exposure of the skin to wash-off formulations can result in suboptimal antimicrobial efficacy (Cutibacterium acnes reduction), exemplifying the need for alternative topical formulations and treatments such as short contact, "leave-on" emollient foams, gels, or creams [3].

Historically, truncal acne has been studied less than facial acne but there has been some progress in recent years, with trials that have evaluated different systemic and topical therapies on the trunk-some with more robust evidence than others. Sarecycline $(1.5 \mathrm{mg} / \mathrm{kg} /$ day $)$ is a once-daily, narrow-spectrum tetracycline for the treatment of moderate to severe acne, which was recently evaluated in two identically designed, randomized, placebo-controlled, 12-week, phase III studies in the USA. The primary endpoint for these trials was Investigator Global Assessment (IGA) and inflammatory and non-inflammatory lesion count reduction on the face. Although these studies were not statistically powered to evaluate the effect of sarecycline on the trunk, the back and chest were also evaluated using IGA scores, providing some beneficial evidence for the treatment of moderate to severe truncal acne [4]. In terms of topical treatments, dapsone $7.5 \%$ gel is an antiinflammatory agent that is commonly used for the treatment of truncal acne in the USA. It has 
been evaluated in one small $(n=20)$, open-label, pilot study, which demonstrated efficacy in patients with moderate truncal acne over 16 weeks [5].

Perhaps the most robust evidence for truncal acne comes from trifarotene $(50 \mu \mathrm{g} / \mathrm{g}$ cream), the first new retinoid molecule for the treatment of acne in 25 years, approved by the US Food and Drug Administration (FDA) and European Medicines Agency (EMA) [6, 7]. Trifarotene is a potent, topical retinoid that selectively targets retinoic acid receptor- $\gamma$. This targeted activity results in low systemic exposure, making it suitable for application on the face and large body surface areas like the trunk. Moreover, we consider the vehicle for trifarotene cream to be easily spreadable, with a suitable texture for truncal application. The trifarotene pivotal studies are the first largescale, randomized, vehicle-controlled trials of a topical acne treatment to evaluate the trunk as an official endpoint using the Physician Global Assessment (PGA) scale ( $N=2420$ patients) [6]. Trifarotene treatment significantly reduced both comedones and inflammatory lesions on the face and trunk over 12 weeks versus vehicle $(p<0.001)$, demonstrating that topical retinoids can be suitable for first-line treatment of truncal acne [6]. Given some dermatologists' perception that retinoids may be unsuitable for the trunk, it is encouraging to see that trifarotene was well tolerated on both the trunk and face, and that the tolerability and safety profile was acceptable and manageable overall [6]. From our practical clinical experience, it seems that trifarotene may be a good option even for those with dry skin or prone to atopic dermatitis.

Acne is a long-term disease, so we must evaluate long-term safety data for any treatment. Unlike most long-term safety trials that include patients from the main phase III efficacy trials, the long-term safety of trifarotene was independently evaluated in a multicenter, open-label study of 453 patients [7]. Therefore, it is very likely that the manageable tolerability and safety profile, and high treatment compliance rates (greater than $95 \%$ on the face and trunk) observed over 1 year are representative of a "true" acne patient population and relevant to clinical practice [7].

The trifarotene pivotal studies demonstrated its efficacy as a monotherapy; in clinical practice, trifarotene could be prescribed alone or in combination with BPO or oral antibiotics, with no contraindications for combining these treatments. Currently, trifarotene is being evaluated in combination with oral doxycycline in a phase IV trial including patients with severe acne, with results expected in 2021 (NCT04451330). However, the selection of combination regimens should depend on acne severity, patient history, suitability, and, importantly, preference. In our experience, many dermatologists avoid prescribing isotretinoin because of the legal implications and responsibility such as the need for written, informed consent and repeated pregnancy testing every 4 weeks. Similarly, a growing number of patients do not wish to use oral isotretinoin because of the pregnancy contraindication and numerous follow-up tests needed for monitoring side effects. Many dermatologists are also reluctant to prescribe oral antibiotics because of the increasing risk of bacterial resistance and because treatment adherence can be low. Therefore, trifarotene represents an important, new treatment option for the trunk, which may reduce superfluous use of antibiotics in acne treatment.

Regardless of the combination regimen that is selected, we must provide adequate guidance to our patients for topical application, including the use of mild or soapless cleansers and moisturizer to minimize skin dryness and irritation. Patients are typically educated/influenced by the media to "spot" treat; however, field treatment is more appropriate for large surface areas of the trunk.

Ultimately, we must be willing to encompass evidence-based alternatives to systemic treatments where possible and seek better topical application approaches for our patients. We must also collectively strive to provide equal importance to both the face and trunk in both clinical research and consultations to provide the most suitable treatments. This will help us prevent the long-term sequelae of acne such as 
scarring and dyspigmentation and relieve the burden of acne on our patients.

\section{ACKNOWLEDGEMENTS}

Funding. Galderma funded the journal's Rapid Service Fee.

Medical Writing and/or Editorial Assistance. Medical writing support was provided by Dr. Arti Sikka (Publicis Langland). This assistance was funded by Galderma.

Authorship. All named authors meet the International Committee of Medical Journal Editors (ICMJE) criteria for authorship for this article, take responsibility for the integrity of the work as a whole, and have given their approval for this version to be published.

Authors' Contributions. Dr. Linda Stein Gold and Professor Thomas Dirschka drafted the manuscript.

Disclosures. Dr. Stein Gold is an investigator and/or consultant and/or speaker for Galderma, Ortho Dermatologics, Sun, Vyne, Sol Gel, Almirall, and Novartis. Professor Dirschka has received research support, and/or lecture fees, and/or is an advisory board member for Almirall, Biofrontera, Dr. Pfleger, Galderma, GSK, Infectopharm, Janssen-Cilag, Leo, Meda, Neracare, Novartis, Riemser, and Scibase.

Compliance with Ethics Guidelines. This article is based on previously conducted studies and does not contain any new studies with human participants or animals performed by any of the authors.

Open Access. This article is licensed under a Creative Commons Attribution-NonCommercial 4.0 International License, which permits any non-commercial use, sharing, adaptation, distribution and reproduction in any medium or format, as long as you give appropriate credit to the original author(s) and the source, provide a link to the Creative Commons licence, and indicate if changes were made. The images or other third party material in this article are included in the article's Creative Commons licence, unless indicated otherwise in a credit line to the material. If material is not included in the article's Creative Commons licence and your intended use is not permitted by statutory regulation or exceeds the permitted use, you will need to obtain permission directly from the copyright holder. To view a copy of this licence, visit http://creativecommons.org/licenses/by$\mathrm{nc} / 4.0 /$.

\section{REFERENCES}

1. Liu C, Tan J. Understanding truncal acne: a practical guide to diagnosis and management. Skin Ther Lett. 2017;12(3).

2. Webster J, Leyden JL, Gross JA. Results of a phase III, double-blind, randomized, parallel-group, non-inferiority study evaluating the safety and efficacy of isotretinoin-lidose in patients with severe recalcitrant nodular acne. J Drugs Dermatol. 2014;13(6):665-70.

3. Leyden JJ. Efficacy of benzoyl peroxide (5.3\%) emollient foam and benzoyl peroxide (8\%) wash in reducing Propionibacterium acnes on the back. J Drugs Dermatol. 2010;9(6):622-5.

4. Moore A, Green LJ, Bruce S, et al. Once-daily oral sarecycline $1.5 \mathrm{mg} / \mathrm{kg} /$ day is effective for moderate to severe acne vulgaris: results from two identically designed, phase 3, randomized, double-blind clinical trials. J Drugs Dermatol. 2018;17(9):987-96.

5. Del Rosso JQ, Kircik L, Tanghetti E. Management of truncal acne vulgaris with topical dapsone $7.5 \%$ gel. J Clin Aesthet Dermatol. 2018;11(8):45-50.

6. Tan J, Thiboutot D, Popp G, et al. Randomized phase 3 evaluation of trifarotene $50 \mu \mathrm{g} / \mathrm{g}$ cream treatment of moderate facial and truncal acne. J Am Acad Dermatol. 2019;80(6):1691-9.

7. Blume-Peytavi U, Fowler J, Kemény L, et al. Longterm safety and efficacy of trifarotene $50 \mu \mathrm{g} / \mathrm{g}$ cream, a first-in-class RAR- $\gamma$ selective topical retinoid, in patients with moderate facial and truncal acne. J Eur Acad Dermatol Venereol. 2020;34(1):166-73. 